\title{
ON A SYMBOLIC CAD FRONT-END FOR DESIGN EVALUATION BASED ON THE PI-THEOREM
}

\author{
STEPHAN RUDOLPH \\ Stuttgart University, Germany
}

\begin{abstract}
The current implementation and theoretical foundation of a possible symbolic front-end for CAD systems for the evaluation of engineering design objects during the design process is described. Based on implicit functional descriptions of the design object (i.e. the design parameters contained in the database of the solid modeler), the Pi-Theorem is used to derive the associated dimensionless groups. Based on the assumed validity of the evaluation hypothesis that "any minimal description in the sense of the Pi-Theorem is an evaluation", these automatically generated dimensionless groups serve then as a symbolic representation for the purpose of design object evaluation.
\end{abstract}

\section{Introduction}

Due to the complexity of problems inherent to the design process of engineering objects, there has been a significant amount of effort to support the designer by means of CAD/CAE systems able to ease many of the designers routine tasks (SDRC, 1993). Since the benefit and gain of productivity of such software systems is widely accepted, the development of software systems with an even greater functionality is an area of intensive current research (ten Hagen et al., 1991; Dym, 1994).

While in many new developments the emphasis lies on the application of new AI-based techniques to the area of design, there has also been a significant effort in the traditional engineering community to formalize the design process from an engineering viewpoint (Pahl and Beitz, 1993; Suh, 1990). Since the design of a technical product involves the definition of its purpose, functional descriptions defined as functional relationships between physical input, output and state variables may be used throughout the design process to represent design object properties independent of a particular solution (VDI, 1987). During the design process, this functional description becomes then more and more concrete (Andreasen, 1992).

Since the formalism of dimensional analysis based on the $\mathrm{Pi}$-Theorem requires only qualitative information about the relevance list of the physical design para- 
meters, this method is most ideally suited for processing qualitative physical knowledge encoded in such functional descriptions of the design object. Dimensional analysis has therefore been already been applied to engineering design problems in the past (Kloberdanz, 1991; Dolinskii, 1990), where it was used to ease the modeling and helped to gain a deeper understanding of the functional behavior of the design object. Other works using dimensional analysis as a basis for the technique of qualitative reasoning (Bhaskar and Nigam, 1990; Sycara and Navichandra, 1989) on design objects have originated from the field of AI and have once more underlined the usefulness of this symbolic technique.

In this work it is shown that dimensional analysis can be used to solve the evaluation problem which frequently occurs during design synthesis when choosing among various design alternatives. Since dimensional analysis relies on the functional modeling of the design object, the applicability of the method is restricted to the concept and limitations of functional modeling in the CAD process (Kuttig, 1993). The implementation of this symbolic method could represent an useful enlargement to existing CAD systems by creating more abstract, symbolic information created from the physical descriptions of the design object contained in the database of the solid modeler. It is important to note that the existing prototype could simply be incorporated into the application programming interface of commercially available CAD/CAE systems (SDRC, 1993) without imposing any changes of the currently valid CAD/CAE paradigms or technologies (Hoschek, 1993; Hoschek and Dankwort, 1994).

To introduce the theoretical concept of the evaluation hypothesis based on dimensional analysis heavily used in the later sections, the evaluation problem of technical design objects as one of the key problems of design analysis and its place in the design process during design synthesis is briefly introduced in section 1 . Section 2 states the key ideas of the evaluation hypothesis. Section 3 contains the necessary proofs and section 4 presents the most important properties which can be derived from these proofs. Section 5 gives two short analytic engineering examples to demonstrate the suggested technique. Section 6 presents the current status of the implementation of the suggested symbolic front-end and closes with an outlook on further conceptual developments.

In the following, the general framework of the design process is described and the evaluation problem inherent to the design process is identified.

\subsection{DESIGN PROCESS}

In a simplified view the design process can be understood as a sequence of more or less related decisions. These decisions affect the selection of a design topology as well as the selection of appropriate sizes of the related design parameters $x_{1}$ to $x_{n}$ which describe the selected topology with sufficient precision.

It is evident that at almost every moment during the design process, design al- 
ternatives have to be evaluated with respect to the design evaluation criteria. How these decisions based on evaluation techniques are made is therefore of crucial importance for the sequence of events in the design process. For this reason, the problems underlying the construction of evaluation models will be investigated further in the following section.

\subsection{EVALUATION PROBLEM}

If one accepts the principle of decomposition of a general goal into several smaller subgoals and the aggregation of evaluations components into a global evaluation respectively, then an evaluation model based on such an assumption can only be a valuable tool for decision making in the design process if at least acceptable answers can be found for the following central questions:

- how to structure the used goal criteria hierarchy?

- how to determine the evaluation of various distinct goal criteria?

- how to aggregate multiple goal criteria into one single goal criterion?

Due to the lack of a formal methodology providing answers to these fundamental questions, most classical decision making models require these questions to be answered by a human decision maker (Hwang and Yoon, 1981). The task of the human decision maker is to establish the description graph of the design object, to determine the evaluation graph and then find the corresponding mapping of the description onto the evaluation. This is shown in Figure 1. The influence of the
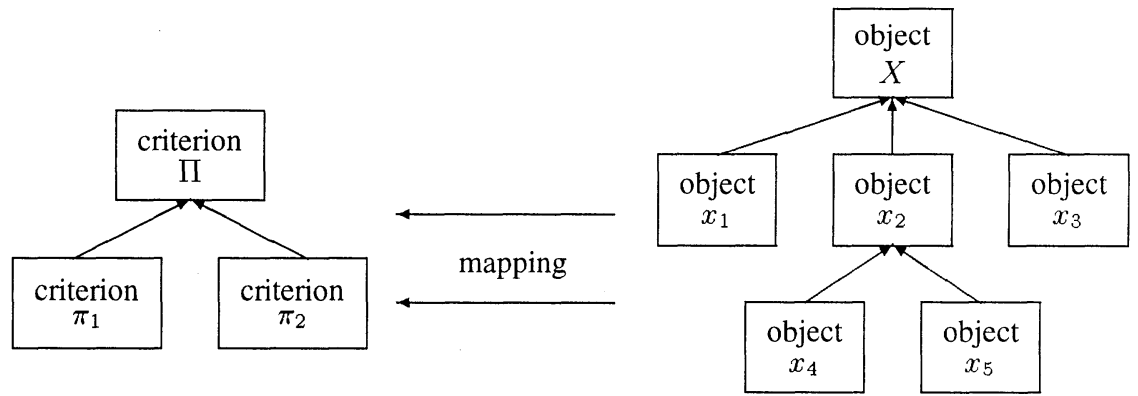

Figure 1. Object description space $X$, mappings and evaluation space $\Pi$.

decision maker leads to the central question of to what extent a decision reflects the personal beliefs of the decision maker or whether an evaluation should be the unique property of the design object. This issue will be discussed in the following section.

\section{Motivation}

From a comparative analysis of some existing decision theories and evaluation models (Rudolph, 1995) it can be concluded that: 
- Under the assumption that an objective evaluation exists in general, it may not depend on the arbitrarily chosen definitions of physical units and therefore has to be dimensionless.

- A reproducible and objective evaluation can only exist if it is based on and derived from some type of law which has to be dimensionally homogeneous.

- An evaluation method should turn into exact physics and be consistent in the case of complete knowledge about a design object.

A universal method to construct dimensionless quantities from dimensionally homogeneous equations is given through the Pi-Theorem, which will be presented in the next section. To ease the understanding of the introduced model, from now on the following terminology will be used as shown in Figure 2, which is essentially the same diagram as shown in Figure 1. In Figure 2 the $x_{i}$ and $X$ represent the description, while $\pi_{j}$ and $\Pi$ represent the corresponding evaluation. The mapping is represented by $\varphi_{0}$ and $\varphi_{j}$, while $\varphi_{1}$ and $\varphi_{3}$ are the aggregation functions of the partial evaluations and the partial descriptions respectively.

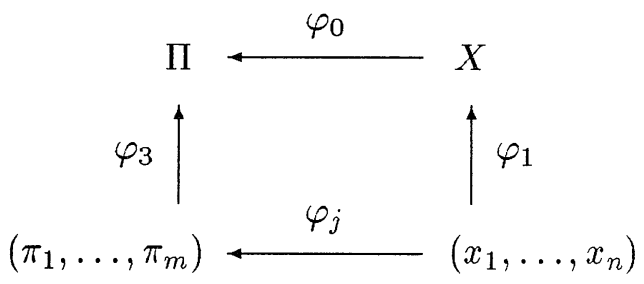

Figure 2. Object description space $X$, mappings $\varphi$ and evaluation space $\Pi$.

\section{Foundation}

Physical quantities may be grouped into the two classes of so called primary and secondary quantities (Bridgman, 1922). Primary quantities are hereby quantities whose reference measurement is one of the base units of the employed unit system. Secondary quantities are derived from primary quantities by some function $f$. In this respect time (measured in $s$ ) and length (in $m$ ) are primary quantities, while velocity (in $\mathrm{m} / \mathrm{s}$ ) is a secondary quantity. Ratios of both primary and secondary quantities are invariant under scale transforms of the type $x^{\prime}=\alpha x$ of physical units, e.g. $1[$ inch $]=0.0254[\mathrm{~m}]$. For the special properties of the function $f$ the so called Product-Theorem can be proven (Bhaskar and Nigam, 1990; Bridgman, 1922):

Product-Theorem. Due to the invariance of ratios of physical quantities under scale transforms of physical units, it can be shown that the function $f$ relating $a$ secondary quantity to some appropriate primary quantities $x_{1}, \ldots, x_{n}$ is of the 
form

$$
f=C x_{1}^{\alpha_{1}} x_{2}^{\alpha_{2}} \cdots x_{n}^{\alpha_{n}}
$$

with $n \varepsilon \mathbb{N}, C$ and the $\alpha_{j i} \varepsilon \mathbb{R}$.

This property of secondary quantities is then used to prove the so called Buckinghamor Pi-Theorem (Bhaskar and Nigam, 1990; Bridgman, 1922):

Pi-Theorem. From the existence of a dimensionally homogeneous and complete equation $f$ of $n$ physical quantities $x_{i}$ the existence of an equation $F$ of only $m$ dimensionless quantities $\pi_{j}$ can be shown

$$
\begin{aligned}
f\left(x_{1}, \ldots, x_{n}\right) & =0 \\
F\left(\pi_{1}, \ldots, \pi_{m}\right) & =0
\end{aligned}
$$

where $r=n-m$ is the rank of the dimensional matrix constructed by the $x_{i}$ and with dimensionless quantities $\pi_{j}$ of the form

$$
\pi_{j}=x_{j} \prod_{i=1}^{r} x_{i}^{-\alpha_{j i}}
$$

with $j=1, \ldots, m \varepsilon \mathbb{N}$ and the $\alpha_{j i} \varepsilon \mathbb{R}$ as constants. (Examples of dimensional matrices are given in the application section.)

An evaluation represents a qualitative and quantitative measure of an object or process. As long as its representation form is still redundant, this redundancy can be eliminated without loss of information. Therefore an evaluation possesses the property of a redundancy-free representation form and is minimal in this respect (Rudolph, 1995). This means that the number of independent parameters can't be reduced any further. This is expressed in the hypothesis that "any minimal description in the sense of the Pi-Theorem is an evaluation".

\section{Evaluation}

Using the proof of the Pi-Theorem, the following list of selected properties can be shown for the evaluation method (Rudolph, 1995):

- Evaluation. The problem of evaluation can be principally reduced to a problem of description. The problem of evaluation is solved exactly in those cases where a complete description exists.

- Minimality. The dimensionless product exponents form a basis in the sense of a linear vector space. The properties of a vector space basis like minimality is therefore also valid for fundamental systems of dimensionless products. 
- Granularity. The addition of one more $x_{n+1}$ to the original description set of $x_{1}, \ldots, x_{n}$ adds one more $\pi_{m+1}$ and leaves the original set of evaluation components $\pi_{1}, \ldots, \pi_{m}$ unchanged. This property supports the experience of hierarchical refinement in the sequence of the design process.

- Hierarchy. Solving $F$ for a specific $\pi_{j}$ creates immediately a consistent hierarchy as shown in Figure 2. This property can be extended to multiple hierarchies.

- Sensitivity. The differential formulation of the model laws with $\pi_{j}=$ const is $d \pi_{j}=0$. Differentiating equation (3) leads to

$$
d \pi_{j}=\frac{\partial \pi_{j}}{\partial x_{j}} d x_{j}+\sum_{i=1}^{r} \frac{\partial \pi_{j}}{\partial x_{i}} d x_{i} \quad j=1, \ldots, m
$$

and setting $d \pi_{j}=0$ leads to the general form of an iso-line of an evaluation component. If only infinitesimal changes of two design parameters $x_{j}$ and $x_{i}$ are permitted, with all other changes equal to zero one obtains

$$
\frac{\partial x_{j}}{\partial x_{i}}=\alpha_{j i} \frac{x_{j}}{x_{i}} \quad \begin{aligned}
& i=1, \ldots, r \\
& j=1, \ldots, m
\end{aligned}
$$

which is analogous to the expression derived in Bhaskar and Nigam (1990) for the purpose of "qualitative reasoning".

- Modularity. The structure of the dimensionless products can be encoded into a topological matrix $a$. With additional use of the unit matrix $I$ the coupling matrix $\kappa=a^{T} a$ is

$$
\kappa=a^{T} I a
$$

This is an analogy to the construction of a stiffness matrix of a structure created from stiffness matrices of multiple finite elements, see also Table 4.

A few ideas on how the method can be tested is given by the fulfillment of the following selected statements and arguments (Rudolph, 1995):

- Causality. The evaluation $\Pi$ is determined by the complete description $X$ in the mathematical sense as a necessary and sufficient condition.

- Invariance. The evaluation $\Pi$ is invariant under scale transforms of the physical units employed in the description $X$ of the object or process.

- Abstraction. Since the evaluation $\Pi$ is the property of a whole class of similar but well distinct objects in $X$, the mapping from $X$ to $\Pi$ is mathematically surjective and not injective.

- Consistency. Since the evaluation is generated by a mapping, the consistency over multiple hierarchy levels is guaranteed if the theory underlying the description hierarchy is consistent. 


\section{Application}

The evaluation method is shown using two examples. The first example focuses on the demonstration of some of the properties of the method like the derivation of the evaluation components and the demonstration of hierarchy and consistency. The second example is taken from a publication in the area of AI (Bhaskar and Nigam, 1990), so that the link established by the mathematical formulation between engineering design evaluation and AI reasoning techniques becomes evident.

\subsection{AUTOMOBILE}

If one tries to evaluate the aerodynamic properties of an automobile like the one sketched out in Figure 3, the relevant physical quantities for the description of the

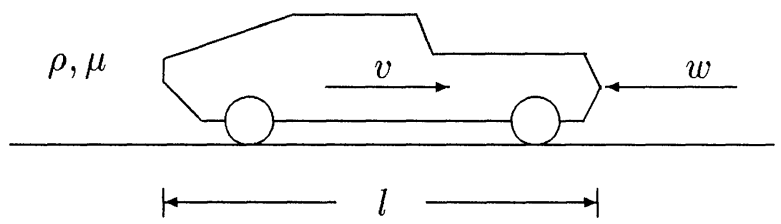

Figure 3. Relevant design parameters of car drag model.

underlying physical process construct the dimensional matrix shown in Table 1. From this dimensional matrix with dimension $n=5$, rank $r=3$ the following

TABLE 1. Dimensional matrix of car drag parameters.

\begin{tabular}{|c||rrr||c|l|}
\hline Symbol ||$[M]$ & {$[L]$} & {$[T]$} & SI-Units & Meaning \\
\hline$l$ & & 1 & & $m$ & characteristic length \\
$v$ & & 1 & -1 & $\mathrm{~m} / \mathrm{s}$ & velocity of car \\
$\rho$ & 1 & -3 & & $\mathrm{~kg} / \mathrm{m}^{3}$ & density of air \\
$\mu$ & 1 & -1 & -1 & $\mathrm{~kg} / \mathrm{ms}$ & viscosity of air \\
$w$ & 1 & 1 & -2 & $\mathrm{~kg} \mathrm{~m} / \mathrm{s}^{2}$ & drag of car \\
\hline
\end{tabular}

$m=n-r=2$ dimensionless products $\pi_{1}$ and $\pi_{2}$ can be derived. According to the

$$
\begin{array}{ll}
\pi_{1}=\frac{w}{\rho v^{2} l^{2}} & \left(\equiv c_{w}\right) \\
\pi_{2}=\frac{v l \rho}{\mu} & (\equiv R e)
\end{array}
$$


complete description $f\left(x_{1}, \ldots, x_{5}\right)=0$, a relation of the form $F\left(\pi_{1}, \pi_{2}\right)=0$ of two dimensionless variables exists. With reference to the general scheme in Figure 2, this interrelation is shown in Figure 4. While $\varphi_{1}$ is given by the chosen

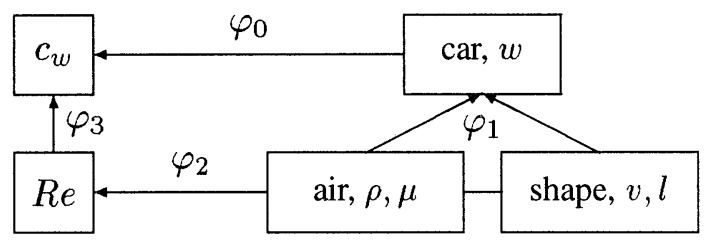

Figure 4. Description and evaluation graph of car drag $w$.

explicit form $w=f(v, l, \rho, \mu)$ of the implicit formulation of $f, \varphi_{0}$ and $\varphi_{2}$ are determined by equation (7) and (8). Thus only $\varphi_{3}$ still needs to be determined. This is mostly done by function approximation of experimental or numerical data for the whole class of geometrically similar objects, as shown in Figure 5 for the class of

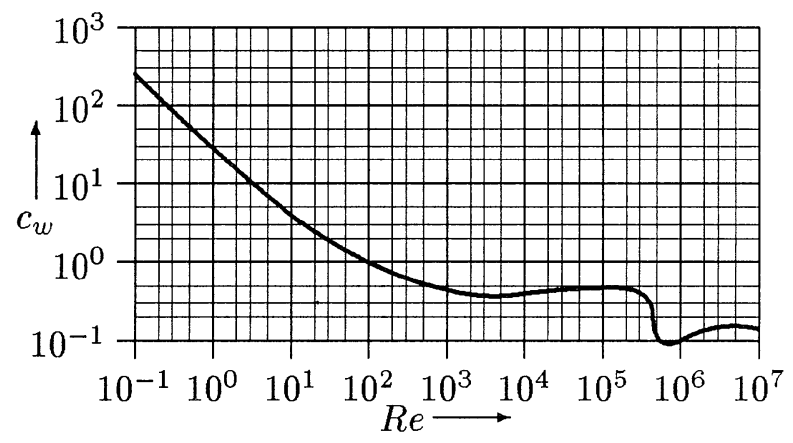

Figure 5. Drag coefficient $c_{w}$ of spheres.

spheres (Zierep, 1972). The approximation and visualization of experimental or numerical data using $\varphi_{3}$ instead of $\varphi_{1}$ is advantageous, since the number of independent parameters is reduced and the interpretation of the obtained relationship more general (Kline, 1986).

\subsection{PRESSURE VALVE}

The modeling of the pressure valve is done according to the presentation in Bhaskar and Nigam (1990). There the whole mechanism as shown in Figure 6 is modeled in two parts: (A) the pipe, using a functional description of $f_{A}\left(\rho, a, p_{i}, p_{o}, q\right)=0$, (B) the orifice, using a functional description of $f_{B}(k, x, p)=0$. The corresponding dimensional matrices are named $\mathrm{A}$ and $\mathrm{B}$, see table 2 . The interaction of both 


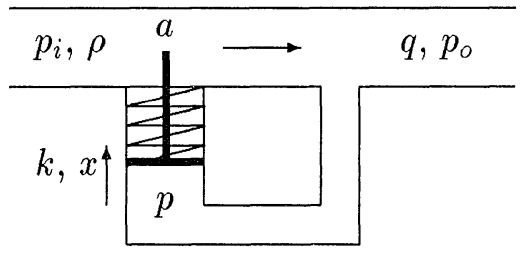

Figure 6. Relevant design parameters of pressure valve.

TABLE 2. Dimensional matrices of pressure valve components A nd B.

\begin{tabular}{|c||rrr||l|l|}
\hline$A$ & {$[\mathrm{M}]$} & {$[\mathrm{L}]$} & {$[\mathrm{T}]$} & SI-units & Meaning \\
\hline$\rho$ & 1 & -3 & & $k g / m^{3}$ & density \\
$a$ & & 2 & & $m^{2}$ & area \\
$p_{i}$ & 1 & -1 & -2 & $N / m^{2}$ & pressure in \\
$p_{0}$ & 1 & -1 & -2 & $N / m^{2}$ & pressure out \\
$q$ & & 3 & -1 & $m^{3} / s$ & stream flow \\
\hline
\end{tabular}

\begin{tabular}{|l||l}
$B$ & {$[\mathrm{M}] \quad[\mathrm{L}] \quad[\mathrm{T}]$}
\end{tabular} \mid SI-units $\mid$ Meaning

\begin{tabular}{|l||rrr||c|l|}
\hline$k$ & 1 & & -2 & $k g / s^{2}$ & spring constant \\
$x$ & & 1 & & $m$ & elongation \\
$p$ & 1 & -1 & -2 & $N / \dot{m}^{2}$ & local pressure \\
\hline
\end{tabular}

components is modeled using $(\mathrm{C})$ two coupling conditions, with functional descriptions $f_{C 1}\left(p, p_{o}\right)=0$ and $f_{C 2}(x, a)=0$. The corresponding dimensional Matrices are named $\mathrm{C} 1$ and $\mathrm{C} 2$, see table 3. Using these four matrices, the following dimensional products can be constructed, which in Bhaskar and Nigam (1990) are also called ensembles

$$
\begin{aligned}
\pi_{1 A} & =\frac{q \rho^{1 / 2}}{a p_{i}^{1 / 2}} \\
\pi_{2 A} & =\frac{p_{0}}{p_{i}} \\
\pi_{1 B} & =\frac{x p}{k} \\
\pi_{1 C} & =\frac{p}{p_{o}} \\
\pi_{2 C} & =\frac{x}{a^{1 / 2}}
\end{aligned}
$$


TABLE 3. Dimensional matrices of functional coupling conditions $\mathrm{C} 1$ and $\mathrm{C} 2$.

\begin{tabular}{|c||ccc||l|l|}
\hline$C 1$ & {$[\mathrm{M}]$} & {$[\mathrm{L}]$} & {$[\mathrm{T}]$} & SI-units & Meaning \\
\hline$p$ & 1 & -1 & -2 & $N / m^{2}$ & local pressure \\
$p_{0}$ & 1 & -1 & -2 & $N / m^{2}$ & pressure out \\
\hline
\end{tabular}

\begin{tabular}{|c|c|c|c|c|c|}
\hline$C 2$ & [M] & [L] & {$[\mathrm{T}]$} & SI-units & Meaning \\
\hline$x$ & & 1 & & $m$ & elongation \\
\hline$a$ & & 2 & & $m^{2}$ & area \\
\hline
\end{tabular}

Since the five ensembles have some of the variables in common, they are not independent from each other and the coupling relationship can be drawn as an undirected graph as shown in Figure 7.

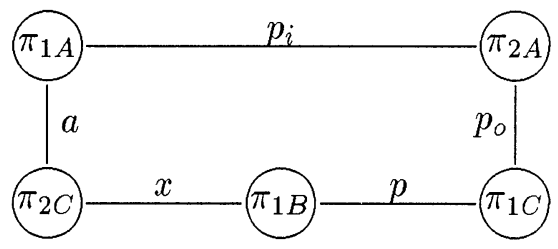

Figure 7. Undirected graph of ensembles (Bhaskar and Nigam, 1990).

With the calculus derived in equation (5), the qualitative behavior of $p_{0}$ due to small changes in $p_{i}$ can now be determined with a strategy called "qualitative reasoning". Using the chain rule, the sign of the derivative $\frac{\partial p_{O}}{\partial p_{i}}$ can be determined, when expanding the derivative along the path $\pi_{1 C} \rightarrow \pi_{1 B} \rightarrow \pi_{2 C} \rightarrow \pi_{1 A}$ in the undirected graph.

$$
\frac{\partial p_{o}}{\partial p_{i}}=\frac{\partial p_{o}}{\partial p} \frac{\partial p}{\partial k} \frac{\partial k}{\partial x} \frac{\partial x}{\partial a} \frac{\partial a}{\partial q} \frac{\partial q}{\partial p_{i}}<0
$$

The coupling matrix $\kappa$ of the design variables constructed using equation (6) depends only on the product form of the dimensionless products and is shown in Table 4.

In analogy to the construction of a stiffness matrix in cartesian coordinates (Argyris and Mlejnek, 1986) of a structure created from stiffness matrices of multiple finite elements in natural coordinates, the five dimensionless variables can be 
TABLE 4. Design coupling matrix $\kappa$.

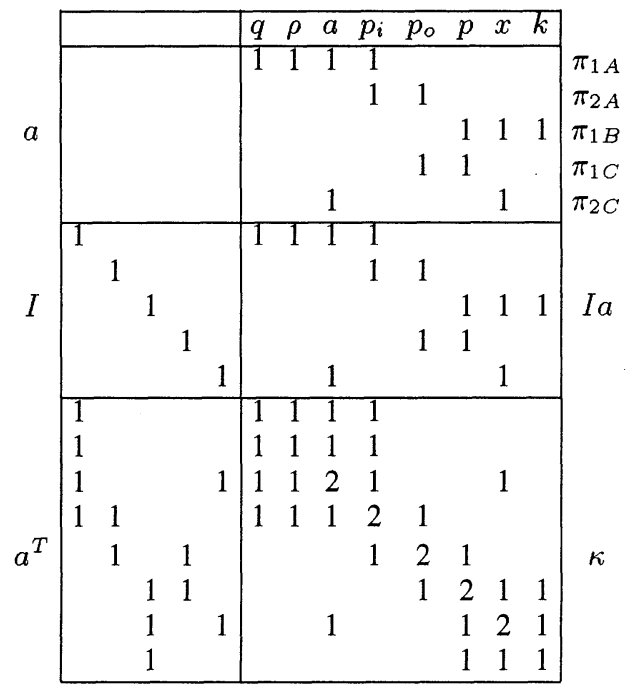

interpreted as the natural degrees of freedom of the design object. In this representation the off-diagonal elements are indicators for the implicit coupling of the design variables.

In the following, a short description of the current implementation of the suggested symbolic CAD-front-end based on the suggested techniques will be given.

\section{Symbolic CAD Front-End}

Using the previous example of the pressure valve design, the currently implemented features of the suggested symbolic front-end for CAD/CAE systems are shown in Figure 8. Since the UNIX-based, network-transparent software programmed in Motif/C is not yet fully incorporated into the CAD application programming interface, a rough sketch of the pressure valve and its physical design parameters involved in the modeling are displayed as a postscript image in the upper right corner. The engineer can then select the appropriate variable descriptions in a database provided by the system, as shown in the upper left corner (see window Database Form).

By selecting the appropriate design variables, the dimensional matrices of the two components of the pressure valve and their topological coupling conditions are constructed (see the three windows Analyze Form), and, according to equation 3, the associated dimensionless groups are automatically generated. The symbolic in- 


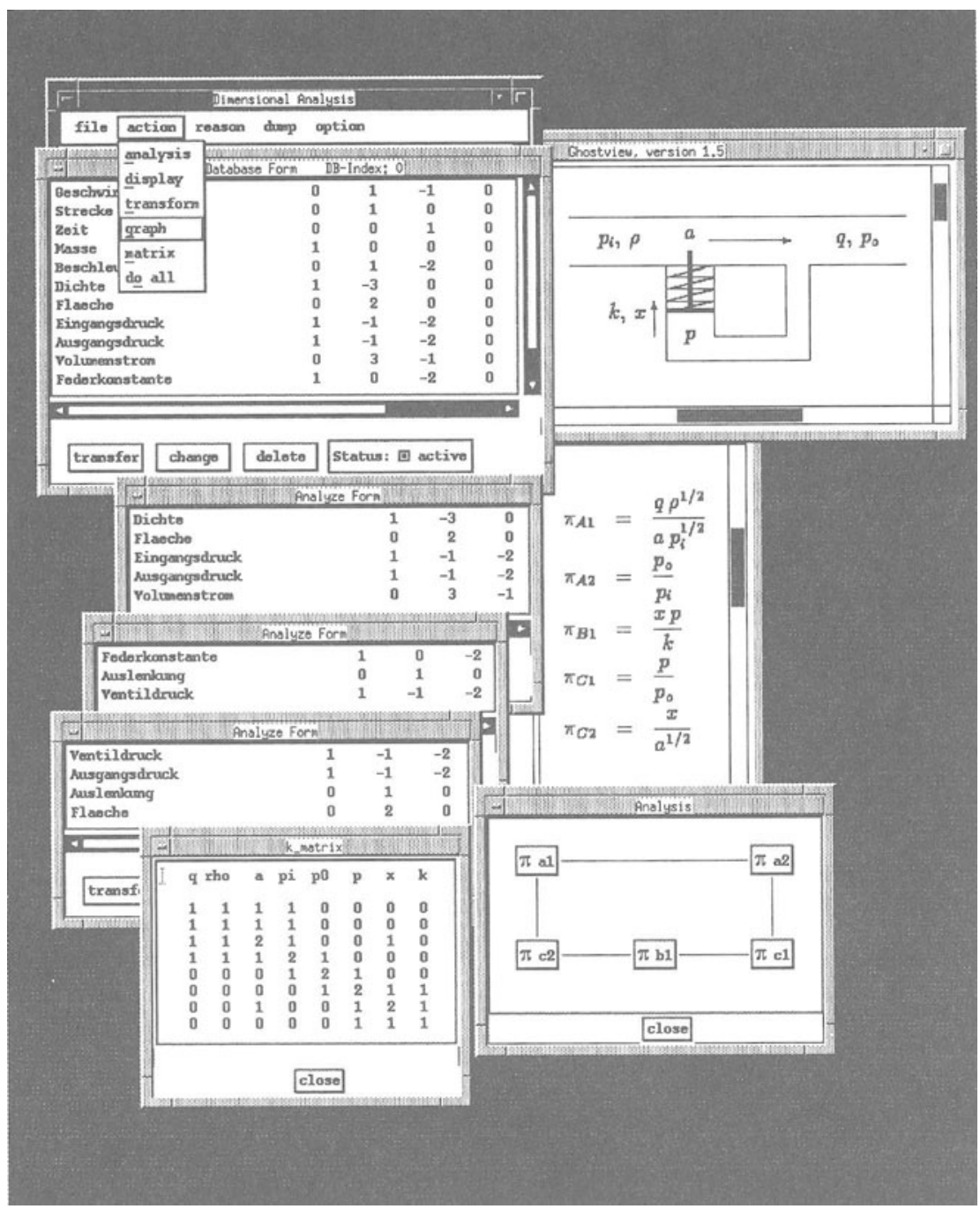

Figure 8. Symbolic CAD front-end prototype.

formation of the building structure of the dimensionless groups is then converted into a postscript format and displayed on the screen as well (see background window in the middle). Using this symbolic information, the coupling matrix according to equation 6 (see window $k \_$matrix) and the corresponding undirected graph (see window Analysis) are also generated by the system.

The manual selection of the design variables and the generation of the symbolic information takes the designing engineer about less than a minute. Once incor- 
porated into the application programming interface, this step can be further automated. This means that it could be imagined that the current design parameter values $x_{1}, \ldots, x_{n}$ are extracted and continuously updated from the database of the solid modeler of the CAD system. The information automatically created by the system in this way would provide the engineer with valuable qualitative insights into the interdependencies of his design. In this respect, the closed loop in the undirected graph window indicates the existence of possible feedback (Bhaskar and Nigam, 1990).

Despite the relative little time of the availability of this software tool and the little experience gained with it until now, the potential of the approach of combining symbolic properties of finite components in analogy to the combination of numerical properties in classical finite element methods seems apparent. Due to the fact that the theoretical basis of the approach relies on the traditional engineering method of dimensional analysis, any other techniques relying on this notation, such as qualitative reasoning techniques (Bhaskar and Nigam, 1990) or engineering design using similar size ranges (Pahl and Beitz, 1993), both based on dimensional analysis, might be straightforwardly added. This concept seems therefore to be worthwhile further investigation.

\section{Conclusion}

By interpretation of the postulated evaluation hypothesis, it is most interesting to note that it can be shown that in a rigorous sense an evaluation is only possible if a complete description in form of functional relationships of the design parameters are known. Since complete functional descriptions are generally difficult to obtain, incomplete descriptions in the case of complex design problems will not allow to evaluate missing technical aspects nor give a complete set of dimensionless products. Therefore not all coupling between design variables will be detected and originally coupled design spaces will be looked at as orthogonal. This fact however is not a specific weakness of the suggested approach, but reflects the fact of incomplete modeling of physical phenomena only. The coupling matrix $\kappa$ in Table 4 will reflect these facts by less off-diagonal entries.

Even though the method put that much emphasis on a clear understanding and a complete description of the problem, dimensional analysis based on the Pi-Theorem has always been a valuable tool for engineers facing and investigating complex technical problems. Proving the completeness of the relevance list $x_{1}, \ldots, x_{n}$ of parameters is restricted to areas of "sharp" physical knowledge but the method with its property as a mathematically necessary condition can't lead to formal contradictions by itself. This imposes no principal restrictions on the use of the method in areas of "unsharp" physical knowledge. 
Of further interest is also the question, what consequences will be caused by the necessary selection of one specific set of $\pi_{1}, \ldots, \pi_{m}$ out of the mathematically equivalent $(n-r)$-parametric solution space of dimensional matrices. Due to the mathematical formulation, the link between classical similarity methods, design evaluation methods and qualitative reasoning techniques could be shown. Such a consistent formulation might ease algorithmic and implementation aspects of future design systems combining these features.

A future design system could possibly exploit detection of similarity for the creation of associations or analogy conclusions (Kodratoff, 1990). Further, the identification of weak coupling of design variables could be used to develop decomposition strategies for automatic design optimization. The investigation of such possibilities might be a first step to make one day more efficient or even more intelligent design support systems a reality.

\section{Acknowledgments}

The author wants to thank Peter Hertkorn for his programming support of the Motif/C based interactive software interface and thanks Robert Schütz for the partial implementation of the underlying algorithms. The financial support of this work by the Deutsche Forschungsgemeinschaft (DFG) and the German interdisciplinary research group Forschergruppe im Bauwesen (FOGIB) "Ingenieurbauten Wege zu einer ganzheitlichen Betrachtung", is greatly acknowledged.

\section{References}

Andreasen, M.: 1992, The theory of domains, Technical Report CUED/G-EDG/TR11, Engineering Department, Cambridge University, Cambridge.

Argyris, J. and Mlejnek, H.-P.: 1986, Die Methode der Finiten Elemente I, Vieweg Verlag, Braunschweig.

Bhaskar, R. and Nigam, A.: 1990, Qualitative physics using dimensional analysis, Artificial Intelligence, 45, 73-111.

Bridgman, P.: 1922, Dimensional Analysis, Yale University Press, New Haven.

Dolinskii, I.: 1990, Use of dimensional analysis in the construction of mechanical assemblies for optical instruments, Soviet Journal of Optical Technology, 57(8), 512-514.

Dym, C.: 1994, Engineering Design. A Synthesis of Views, Cambridge University Press, Cambridge.

Hoschek, J. (ed): 1993, Was CAD-Systeme wirklich können, Teubner, Stuttgart.

Hoschek, J. and Dankwort, W. (ed): 1994, Parametric and Variational Design, Teubner, Stuttgart.

Hwang, C.-L. and Yoon, K.: 1981, Multiple Attribute Decision Making, Lecture Series in Economics and Mathematical Systems, Springer-Verlag, Berlin.

Kline, S.: 1986, Similitude and Approximation Theory, Springer-Verlag, New York.

Kloberdanz, H.: 1991, Rechnerunterstützte Baureihenentwicklung, Fortschrittsberichte Reihe 20, Nummer 40, VDI-Verlag, Düsseldorf.

Kodratoff, Y.: 1990, Combining similarity and causality in creative analogy, Proceedings ECAI-90, pp. 398-403. 
Kuttig, D.: 1993, Potential and limits of functional modelling in the CAD process, Research in Engineering Design, 5, 40-48.

Pahl, G. and Beitz, W.: 1993, Konstruktionslehre, Springer-Verlag, Berlin.

Rudolph, S.: 1995, Eine Methodik zur systematischen Bewertung von Konstruktionen, VDI Fortschrittsberichte, Reihe 1, Nummer 251, VDI-Verlag, Düsseldorf (In German. An English version entitled: A methodology for the systematic evaluation of engineering design objects is available on request by email to rudolph@isd.uni-stuttgart.de.

SDRC Master Series: 1993, User's Guide: Model Solution and Optimization, Structural Dynamics Research Corporation, Milford, Ohio.

Suh, N.: 1990, The Principles of Design, Oxford Press, New York.

Sycara, K. and Navinchandra, D.: 1989, Integrating case-based reasoning and qualitative reasoning in engineering design, Proceedings Applications of AI in Engineering, pp. 231-250.

ten Hagen, P. and Tomiyama, T.: 1991, Intelligent CAD Systems I, Springer-Verlag, New York.

VDI Guideline-2221: 1987, Systematic Approach to the Design of Systems and Products (trans. of the German edn 11/1986), VDI-Verlag, Düsseldorf.

Zierap, J.: 1972, ̈̈hnlichkeitsgesetze und Modellregeln in der Strömungslehre, Braun Verlag, Karlsruhe. 